\title{
Modified SURF applied in Remote Sensing Image Stitching
}

\author{
Jianxia Wang and Yawei Wang \\ School of Information Science and Engineering, Hebei University of Science and \\ Technology, Shijiazhuang, 050018 \\ wang_jianxia@126.com,yaweiwanghb@163.com
}

\begin{abstract}
With the development of satellite technology, the application of remote sensing images becomes more and more significant. The coverage of a single remote sensing image is limited, so remote sensing images should be stitched into a larger image by overlapping region. SURF is a commonly algorithm for image mosaic, but the calculation of this algorithm is too heavy. Thus, we propose the method of modified SURF applied in remote sensing image stitching. Firstly, we extract basic feature points by Hessian matrix. Secondly, we improve the accuracy of feature point matches by use of FLANN algorithm and RANSAC. Finally, we take advantage of the algorithm of overlapping region weighted average to blend remote sensing images. Through the experiment, we can prove that the image stitching system can implement remote sensing image mosaic, which enables the smooth transition for image blending and has superiority and efficiency about image stitching.
\end{abstract}

Keywords: remote sensing image, SURF, stitching, RANSAC, weighted average

\section{Introduction}

Image mosaic technology [1] is a commonly used image processing method now. With the development of satellite technology, the application of remote sensing images becomes much widespread. Remote sensing images have applied for the domains of agriculture and natural disaster [2]. Digital maps and satellite photos are produced by image mosaic technology. While the coverage of a single remote sensing image is limited, we form a unique degree of high resolution remote sensing images by stitching overlapping regions. Because the acquisition time and environment of remote sensing images are different, so the key of image stitching is how to accurately select the same feature points of each image.

The technologies of image stitching mainly consist of image registration and image fusion [3]. Because the emphasis of image registration is selecting the feature points, the key of this paper is filtering the error matching points. The methods of image registration include block matching, direct matching and feature points matching which is the most common method. The common algorithms of feature point matching are SIFT [4] and SURF [5]. SIFT algorithm can deal with the changes of translation, rotation, illumination and perspective, then, it has the stability of feature extraction. However the processing of SIFT is complex and the speed of detecting and matching image features is low. Thus Bay has proposed SURF algorithm which identifies gradient patch by use of PCA (principle component analysis) [6] instead of histogram [7]. The local descriptor of PCA takes advantage of image deformation and rotation robustness. The main challenges on image mosaic based on SURF algorithm are as follows: we select the method of detecting basic feature points. Feature vector from SURF is three-dimensional space and matched by the algorithm of nearest neighbor distance. The process of matching is equivalent to calculate the most adjacent search problem for the high dimension space, which needs a complex calculation. Image fusion is faced with the challenges of feature points, perspective and adjustment of color blending. 
In this paper, we implement the stitching of remote sensing images by modified SURF. Firstly, we extract basic feature points by use of Hessian [8]. Secondly, we take advantage of FLANN (fast approximate nearest neighbor search algorithm) [9] to match feature points, which can improve the accuracy and efficiency for this system. Thirdly, we remove the mismatches by RANSAC (Random Sample Consensus) [10] algorithm. Finally, we stitch remote sensing images based on the algorithm of overlapping region weighted average.

The rest of this paper is organized as follows. Section 2 introduces the algorithms of SURF and SIFT. Section 3 describes the principle and process of modified SURF algorithm. Section 4 describes the step of image registration and robust image fusion technology. Section 5 introduces the experiment and result of remote sensing image stitching in this paper. Section 6 describes the conclusion and future work in this paper.

\section{Preparation}

SIFT [4] is an algorithm about partial feature of descriptor proposed by David Lowe in 1999, which has been further improved and developed in 2004. SIFT can process the translation, rotation and affine transformation of images that has the strong capacity to match feature points. SIFT is a kind of algorithm which only take advantage of grayscale properties while ignores the information on color.

SURF [5] algorithm is an improvement of SIFT, mainly reflected in speed and higher efficiency. The main difference between SURF and SIFT is the method of building multi-scale space. SURF can process multi-scale space images at the same time so that it doesn't sample once again, which improves the performance of image stitching.

The feature vector from SURF is three-dimensional space and matched by use of the nearest neighbor distance algorithm. The matching process is equivalent to calculate the most adjacent search problem for the high dimension space which needs a complex calculation. Reference [11] compares some image mosaic algorithms with each other and finds that the problem of nearest neighbor search in high dimensional space can take advantage of the method of k-mean [12] tree and KD-tree [13] which have the performance of image matching and implement FLANN based on the accuracy of user's input and choice of high-dimensional data automation. Thus this paper adopts FLANN to match feature points. In this paper, we take advantage of RANSAC method to improve the accuracy of selecting feature points. Before the step of blending, we rotate the image to the right angles and coordinates by use of perspective transformation method.

In this paper, we adopt the algorithm of overlapping region weighted average to blend images. Firstly, according to the actual situation of the remote sensing image acquisition, we take the overlap region of two images for the process of gray histogram equalization. Then we take advantage of weighted average method to improve the color of image which can gain a scale remote sensing image of higher resolution. Finally, we compare the results of modified SURF and SIFT algorithm, which shows that the algorithm of our paper has superiority and efficiency about remote sensing image stitching.

\section{Algorithm of modified SURF}

\subsection{Building the Hessian Matrix}

Hessian matrix is proposed by Ludwig Otto Hesse which describes the second partial derivative and the local curvature for multivariate function. In SURF, pixel is usually expressed as $l(x, y)$, then it chooses the function of second-order Gaussian as filter which can remove the independence of feature points. By use of the convolution of specific cores we can calculate the second-order partial derivative, thereby the equation of Hessian matrix can be shown as (1): 


$$
H(x, \sigma)=\left[\begin{array}{ll}
L_{x x}(x, \sigma) & L_{x y}(x, \sigma) \\
L_{x y}(x, \sigma) & L_{y y}(x, \sigma)
\end{array}\right]
$$

where $L_{x x}(x, \sigma), L_{x y}(x, \sigma), L_{y y}(x, \sigma)$ are the second-order partial derivative for the pixel of $x$.

We use the discriminant of Hessian matrix to classify all points, then according to the plus or minus about the value of discriminant decide whether this point is the local extremum. The discriminant of Hessian matrix is calculated as equation (2):

$$
\operatorname{det}(H)=\frac{\partial^{2} f}{\partial x^{2}} \frac{\partial^{2} f}{\partial y^{2}}-\left(\frac{\partial^{2} f}{\partial x \partial y}\right)^{2}
$$

In the algorithm of SURF, each point should be compared with other 26 points which are distributed in three-dimensional space. If the value of this point is maximum or minimum, it will be saved as feature points. In the process of detecting feature points, it should take advantage of the corresponding filter. The method of comparison is shown as Figure 1, where $x$ is a point for detecting.

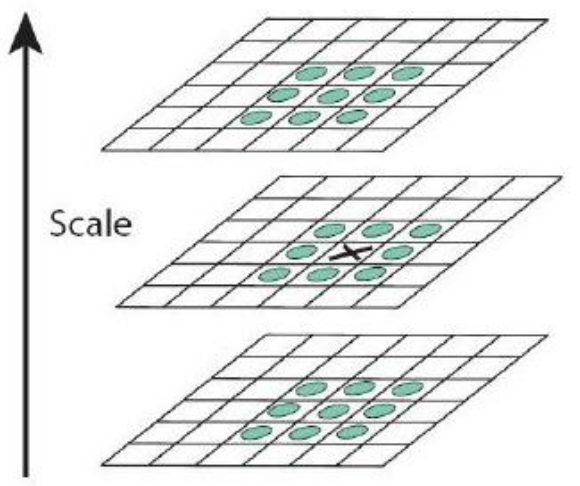

Figure 1. Point for Detecting

\subsection{Process of matching}

Table 1. Descriptor of RANSAC

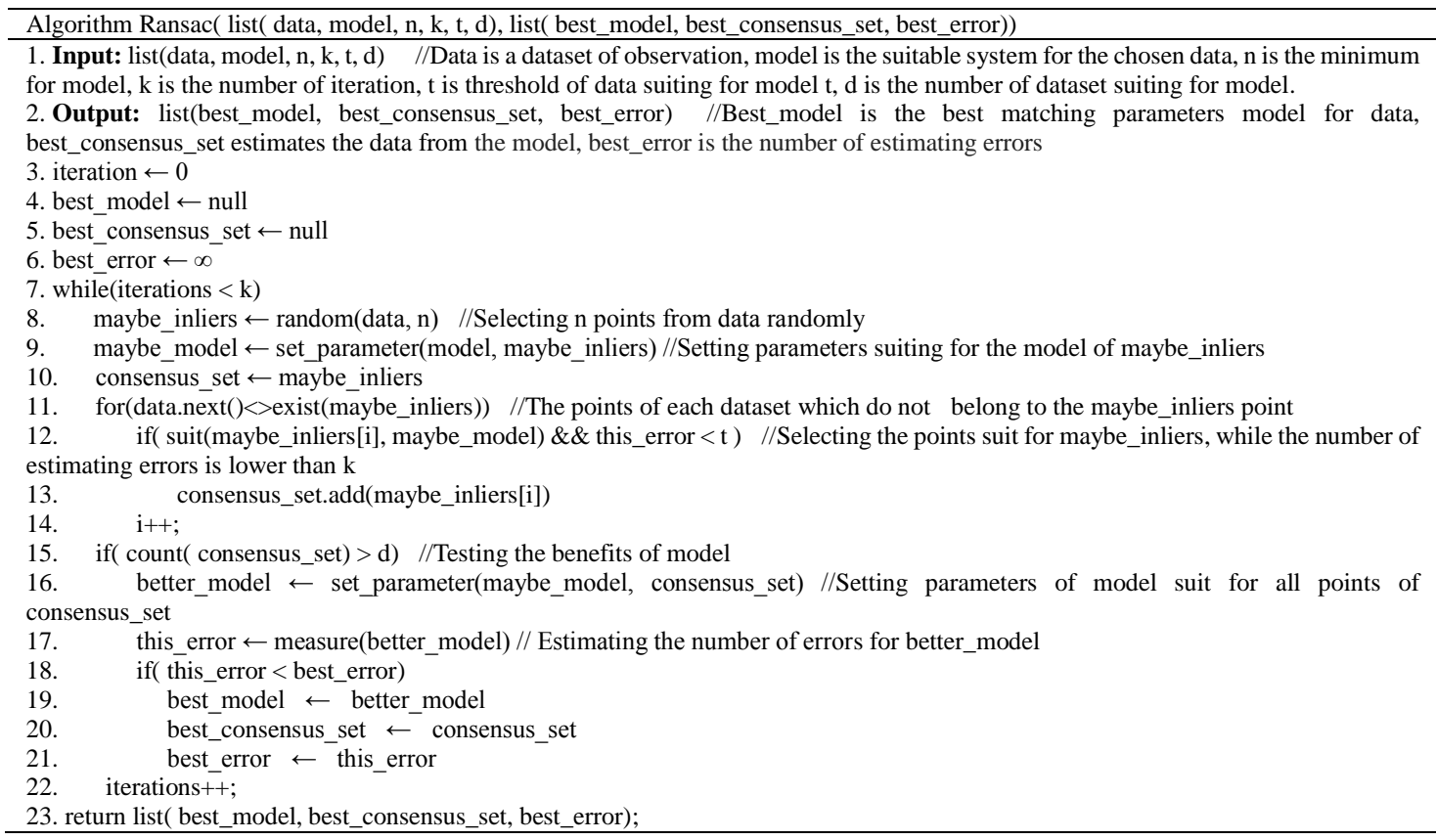


After extracting invariant scale features, we get potential feature matches by use of FLANN method, and remove the mismatches with RANSAC algorithm. RANSAC can estimate a mathematical model by the way of iteration from the dataset which contains the incorrect feature descriptors. It achieves a reasonable result according to specific probability, in our experiment it takes 0.5 by the theory of Lowe. Based on the algorithm of RANSAC in Table 1, we can demonstrate that when the system finds a better model whose error rate is smaller enough, this process will jump the loop and return the result of model.

\section{Image Registration and Fusion}

In this section, we explore the next steps: image registration and fusion for image stitching. It shows the selected algorithms of image registration and fusion.

The purpose of image registration is to transform images into the same angle and coordinates for perspective. In our work, we take advantage of perspective transformation as the image registration algorithm which shows a good performance in this field. This method rotates the images to the same angle and coordinates so that they can be projected in a new viewing plane. There are two steps in the method of perspective transformation: firstly, transform the points of frustum to the homogenous clip space of CVV (Canonical View Volume). Secondly, execute the method of perspective division after the clipping of CVV. According to the above steps, we can get the result in Figure 2. Perspective transformation has solved the deviation problem causing by different shooting angles preliminarily.

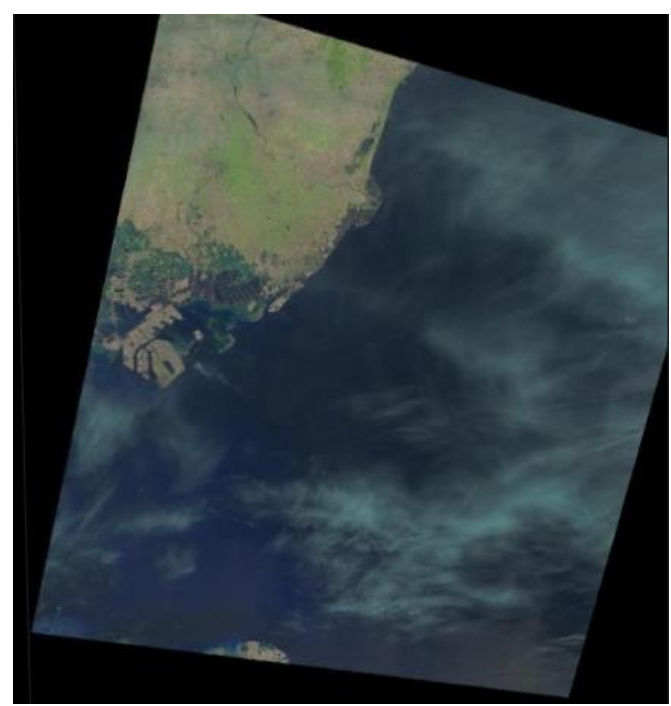

\section{Figure 2. Result of Perspective Transformation}

We deal with the overlapping region of stitching image using the method of histogram equalization. At the actual processing of transformation, we make statistical analysis for the gray scale and calculate the histogram of source images, then map the gray scale to cumulative frequency distributing graph. The equation of this method is shown in (3):

$$
s_{k}=\sum_{j=0}^{k} \frac{n_{j}}{n} \quad k=0,1,2, \cdots, L-1
$$

where $n$ is the sum of pixels from each image, $n_{j}$ is the number of pixels at present, $L$ is the amount of gray scale whose range number is 0 to 255 . Repeating the above steps, we can obtain the mapping relationship between the grayscale of target images and the grayscale of all source images. The method of histogram equalization can enlarge the luminance of image so that the local contrast can be enhanced instead of the holistic express. We can get the gray histogram in Figure 3 and the gray histogram of equalization in Figure 4 from 
original source image. By the comparison of Figure 3 and Figure 4, we can demonstrate that the gray histogram of equalization can enhance the luminance of source image.

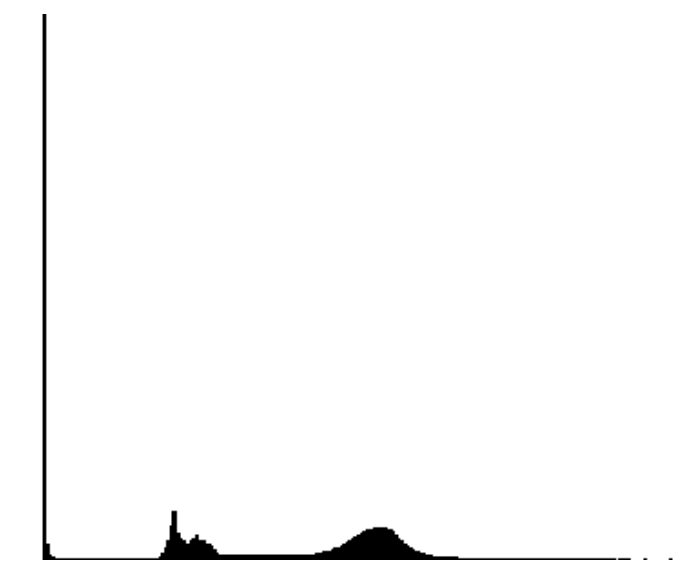

Figure 3. Gray Histogram of Source Image

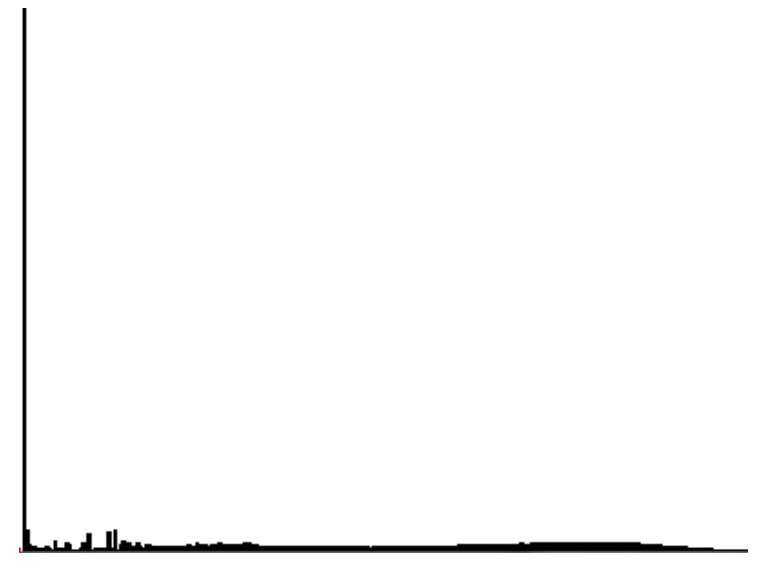

Figure 4. Gray Histogram of Equalization

Generally speaking, image blending is nothing more than a type of interpolation. The goal of the blending is to produce a perfect image where no transition can be seen between the original source images. Weighted average method is a common method to employ the average of images in the overlapping regions. Firstly, we take advantage of the interest region in each source image, whose coordinate of pixel is from its upper-lift corner. The interest region of remote sensing image is shown in Figure 5. The weighted average method is formed as equation (4):

$$
\text { pixel }=\frac{d 1}{d 1+d 2} * \text { pixelImg } 1+\frac{d 2}{d 1+d 2} * \text { pixelImg } 2
$$

where $\mathrm{d} 1$ and $\mathrm{d} 2$ are the distances from the pixel of overlapping region to its upper-lift corner for the two images. The transitional areas between images will hardly visible. The comparison results can be seen directly in the next section. Weighted average method ensures smooth transitions between images despite illumination differences, while preserving high frequency details, so we choose this method in our paper.

\section{Experiments and Results}

The image stitching process is shown in Figure 6. There are two steps in our work: matching and blending. In the part of matching, firstly, our modified SURF is used to detect 
feature points and feature descriptors are 64 dimensional vectors in our experiment. Secondly, among these descriptors, we get potential feature matches by using FLANN method. Lastly, RANSAC is used to estimate a model of consensus set that minimizes matching error rate. This is an iterative process whose goal is to find the largest feature points for transformation. In the part of blending, firstly, perspective transformation has preliminarily solved the deviation problem causing by different shooting angles, in other word, the images has been transformed into the corresponding image in a same coordinate system by perspective transformation. Then, weighted average method is used to make the transformation error or the seam invisible by resetting the value of overlapping region according to equation.

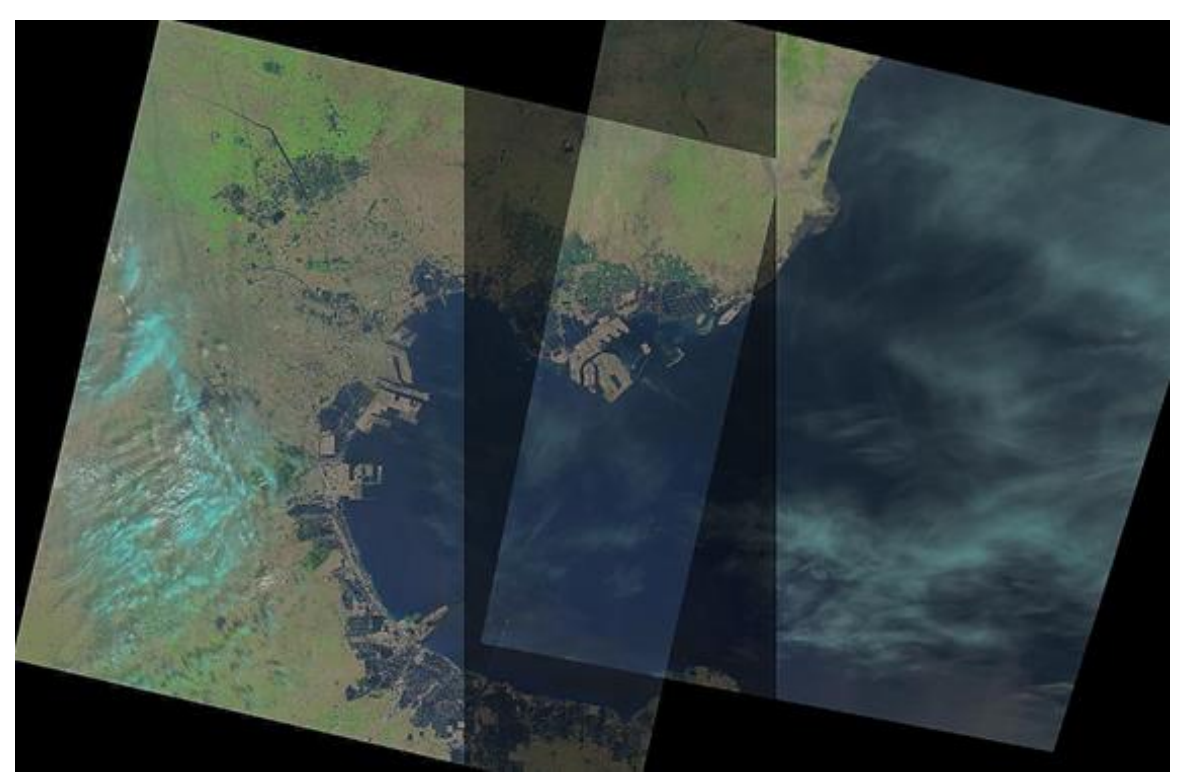

Figure 5. Interest Region of Remote Sensing Images

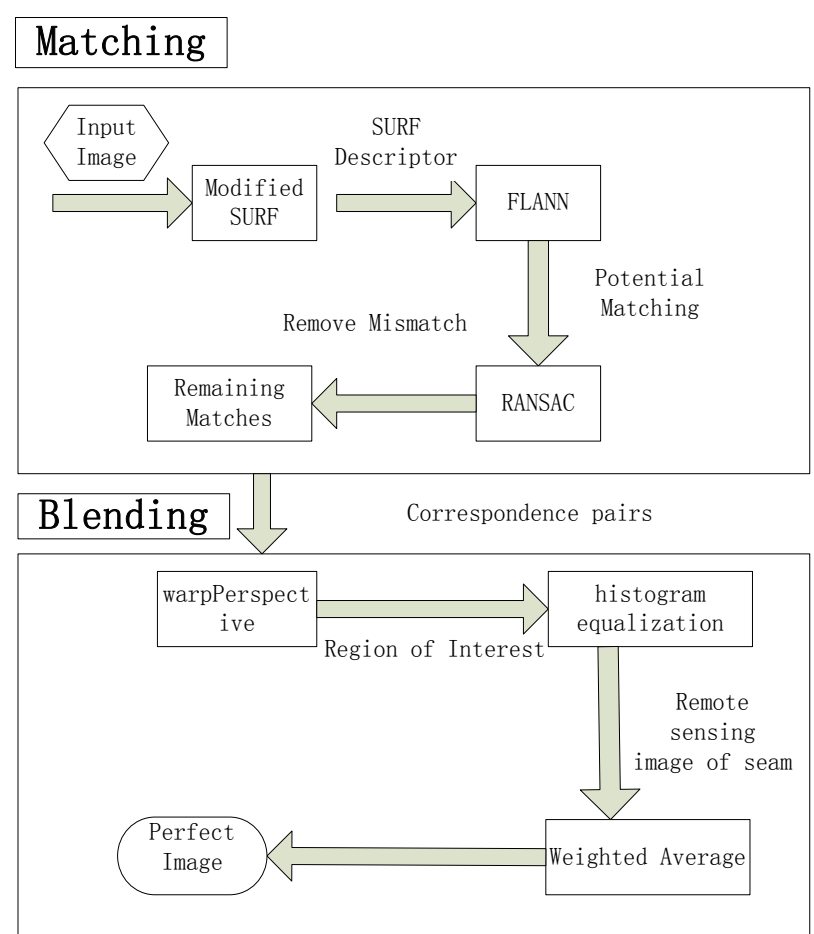

Figure 6. Flow Diagram of Remote Sensing Image Stitching System 
The data of experiment comes from the coastal images filmed by Landsat. The experiment has been done on the platform of visual studio $2010 \mathrm{C}++$ with the library of OpenCV (Open Source Computer Vision Library). In this section, we will compare the result of modified SURF with the result of SIFT. Figure 7 shows the result of remote sensing images stitched based on modified SURF and Figure 8 shows the result of remote sensing images stitched based on SIFT. We can see that the performance of our present stitching process shows better than SIFT demo. The present system can make the seam of stitching image invisible.

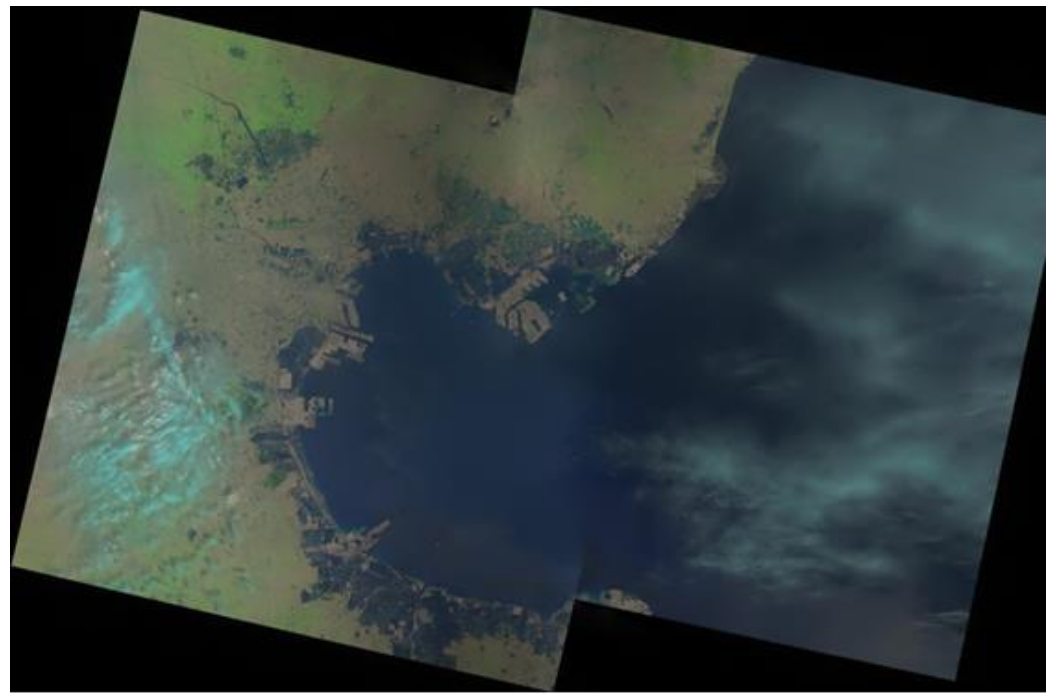

Figure 7. Remote Sensing Images Stitched based on Modified SURF

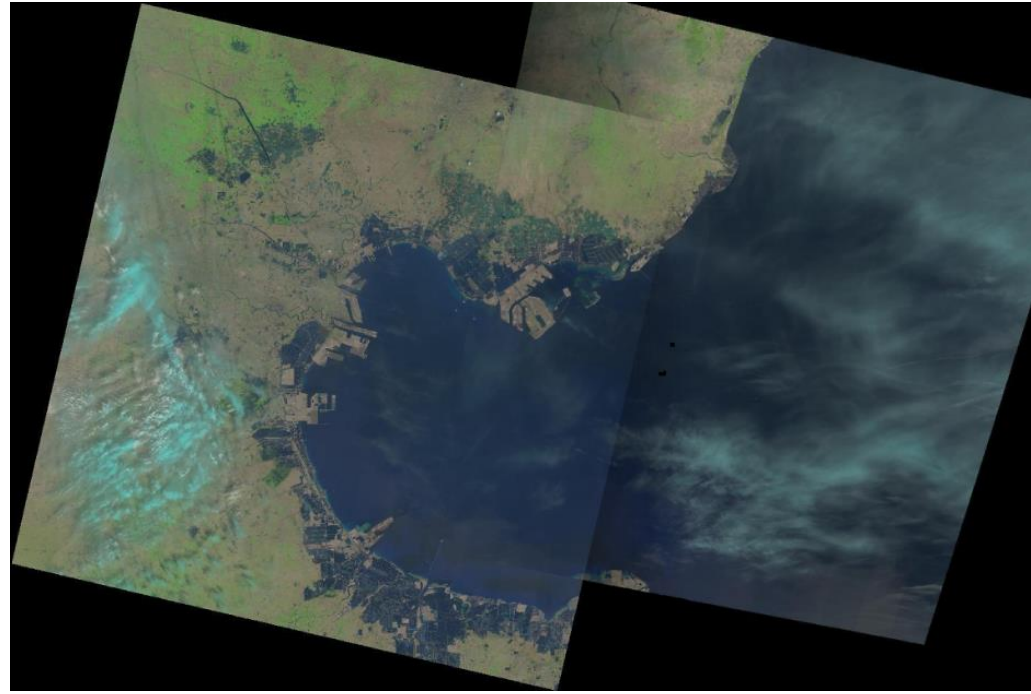

Figure 8. Remote Sensing Images Stitched based on SIFT

\section{Conclusion and Future Works}

Experiments showed that the present remote sensing image stitching process has a good performance. The reasons are as follows: modified SURF algorithm can be created in case there are some changes of illumination, color, blur and et cetera, and processed fast. FLANN and RANSAC improve the repeatability of matching. Perspective transformation and weighted average make the panorama seamless. But the present system shows its 
defects that the system only can stitch two images. So as a future work, we plan to do more research in the area through large data set of remote sensing images.

\section{Appendix}

The test images of this paper are shown in this appendix, which include two coastal remote sensing images filmed by Landsat. The images are in the same size with 1004x1024 resolutions.
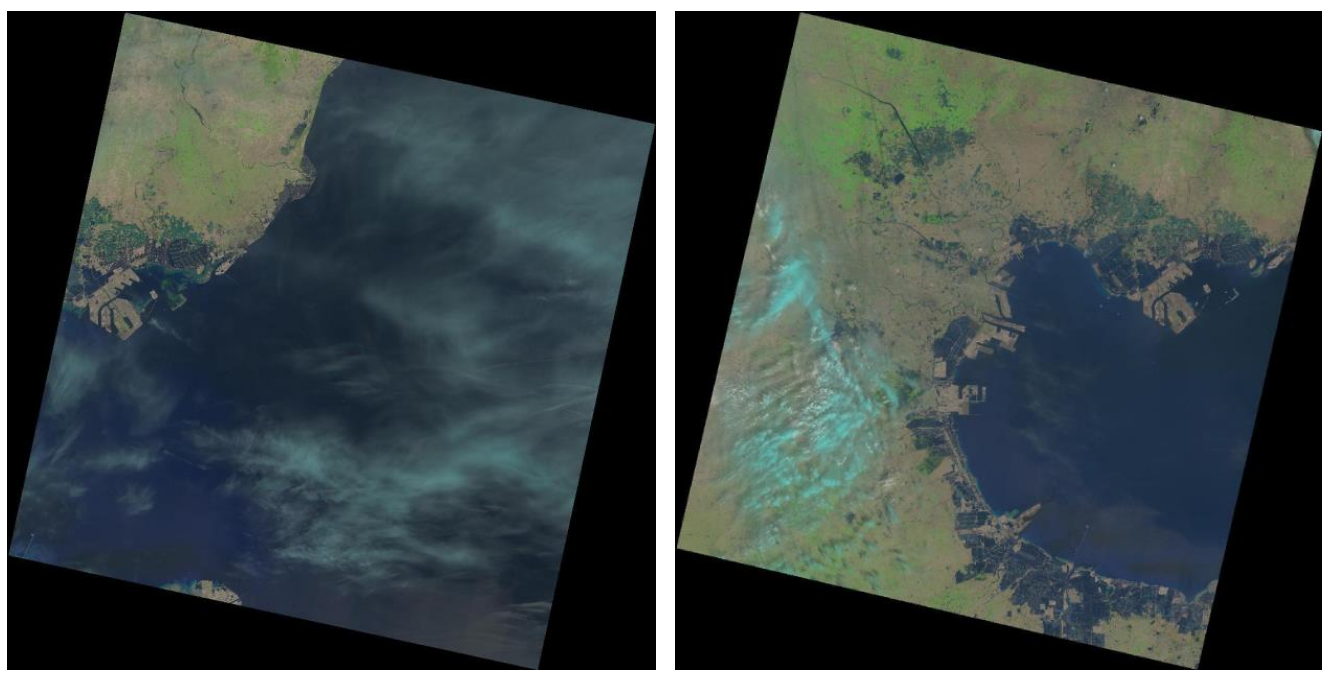

\section{References}

[1] Y. Yang, Y. Gao, H. Li and Y. Han, "An Algorithm for Remote Sensing Image Mosaic Based on Valid Area”, Image and Data Fusion (ISIDF), 2011 International Symposium on, (2011) pp. 1-4.

[2] E. C. Barrett, "Introduction to environmental remote sensing", Routledge, Los Angeles (2013).

[3] T. Stathaki, "Image fusion: algorithms and applications", Academic Press, Los Angeles (2011).

[4] W. L. Zhao and C. W. Ngo, "Flip-Invariant SIFT for Copy and Object Detection", Image Processing, IEEE Transactions on, vol. 22, no. 3, (2013), pp. 980-991.

[5] H. Zhang and Q. Hu, "Fast image matching based-on improved SURF algorithm", Electronics, Communications and Control (ICECC), 2011 International Conference on, (2011), pp. 1460-1463.

[6] H. Xu, C. Caramanis and S. Sanghavi, "Robust PCA via Outlier Pursuit", Information Theory, IEEE Transactions on, vol. 58, no. 5, (2012), pp. 3047-3064.

[7] D. Phan, C.-M. Oh, S.-H. Kim, I.-S. Na and C.-W. Lee, "Object Recognition by Combining Binary Local Invariant Features and Color Histogram", Pattern Recognition (ACPR), 2013 2nd IAPR Asian Conference on, (2013), pp. 466-470.

[8] Hessian matrix, http://en.wikipedia.org/wiki/Hessian_matrix, (2014).

[9] D. Aiger, E. Kokiopoulou and E. Rivlin, "Random Grids: Fast Approximate Nearest Neighbors and Range Searching for Image Search”, Computer Vision (ICCV), 2013 IEEE International Conference on, (2013), pp. 3471-3478

[10] O. Chum and J. Matas, "Optimal Randomized RANSAC", Pattern Analysis and Machine Intelligence, IEEE Transactions on, vol. 30, no. 8, (2008), pp. 1472-1482.

[11] SIFT SURF- E. Rublee, V. Rabaud, K. Konolige and G. Bradski, "ORB: An efficient alternative to SIFT or SURF”, Computer Vision (ICCV), 2011 IEEE International Conference on, (2011), pp. 2564-2571.

[12] KNN- H. Yigit, "A weighting approach for KNN classifier", Electronics, Computer and Computation (ICECCO), 2013 International Conference on, (2013), pp. 228-231.

[13] KD TREE- P. Sirait and A. M. Arymurthy, "Cluster centres determination based on KD tree in K-Means clustering for area change detection", Distributed Framework and Applications (DFmA), 2010 International Conference on, (2010), pp. 1-7. 


\section{Authors}

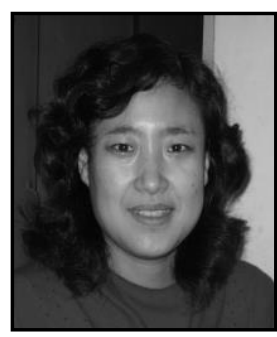

Jianxia Wang, Female, she was born on 1970, received the Master degree in Measurement Technology and Instrument from Yanshan University of China in 2003. Now, she is a professor in the School of Information Science and Engineering at Hebei University of Science and Technology, China. Her main research interests include Network and Database, Pattern recognition.

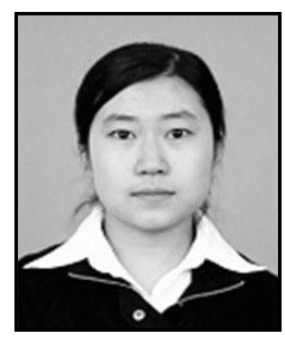

Yawei Wang, Female, she was is born on 1989. She is master in School of Information Science and Engineering at Hebei University of Science and Technology. Her main research interests include Digital Image Processing and Pattern Recognition. 
International Journal of Signal Processing, Image Processing and Pattern Recognition Vol.8, No.8 (2015) 